CHAPTER 2

\title{
Photogrammetry: What, How, and Where
}

\author{
Hafizur Rahaman \\ Faculty of Humanities, Curtin University \\ hafizur.rahaman@curtin.edu.au
}

\begin{abstract}
Developing 3D digital models of artefacts, monuments, excavations, and historic landscapes as part of digital documentation is becoming commonplace in the fields of heritage management, virtual tourism, immersive visualisation, and scientific research. Such 3D reconstruction or 3D data acquisition from a laser scanning process involves high costs, manual labour, and substantial expertise. On the other hand, image-based 3D modelling photogrammetry software offers a comparatively inexpensive alternative and can handle the task with ease. Besides, documenting heritage artefacts with free and open-source software (FOSS) in supporting photogrammetry is getting popular for quality data production.

Due to the present pandemic situation and social distancing restrictions, gallery, library, archive, and museum (GLAM) industries are facing an incremental burden on both their income and visitor traffic, which is affecting their survival. As a way out, we can see some GLAM institutes are trying to expand their collections on digital platforms for showcasing and promoting virtual visits. Numerous online portals and repositories are evolving for archiving, sharing,
\end{abstract}

How to cite this book chapter:

Rahaman, H. 2021. Photogrammetry: What, How, and Where. In: Champion, E. M. (ed.) Virtual Heritage: A Guide. Pp. 25-37. London: Ubiquity Press. DOI: https: //doi.org/10.5334/bck.d. License: CC-BY-NC 
and trading 3D models are also evolving to support this digital 'vibe. This chapter explains the basics of photogrammetry and its development workflow, including data acquisition (photo shooting), data processing, and a few postprocessing tools.

\section{Introduction}

In areas such as heritage documentation, virtual recreation, simulation, crime scene analysis, urban project planning, augmented/virtual/mixed reality, serious games, and scientific research, 3D digital models of artefacts, buildings, archaeological excavation, and historic natural landscapes are becoming increasingly popular. Applications such as Maya, Blender, or 3D Studio Max, which follow traditional geometry-based modelling methods, involve a steep learning curve and require significant time and energy. However, advances in hardware (laser scanners, unmanned aerial vehicles, etc.) and software, have increased the opportunities for the virtual reconstruction of 3D scenes.

Lidar, also called 3D laser scanning, measures distances (ranging) by illuminating the target with laser light and measuring the reflection with a sensor. One can often refer to this pathway as the most suitable for providing the most accurate $3 \mathrm{D}$ models (depending on the environment and the $3 \mathrm{D}$ scanner). However, laser scanners and structured lighting systems are often expensive and require a high level of expertise. Furthermore, this technology has limitations regarding rendered material properties and environmental conditions, for example, dealing with strong sunlight (Nguyen et al. 2012).

There is an urgent need for tools and supports that enable non-expert users to build $3 \mathrm{D}$ reconstruction models comfortably and efficiently, especially for 3D digital documentation and heritage visualisation purpose. In supporting this demand, commercial software packages as well as free and open-source software (FOSS) based on image-based modelling (IBM) or photogrammetry have emerged. Regard3D, Colmap, VisualSfm, and Python Photogrammetry Toolbox are some leading examples of FOSS.

The image-based 3D modelling/reconstruction method uses uncalibrated photographs from cameras and can generate a $3 \mathrm{D}$ point cloud via various algorithms. A certain number of quality photographs, therefore, are required to allow the software to process, match, and triangulate visual features and to further generate the 3D point-cloud data. Structure from motion (SfM) is one of the most popular techniques in the image-based modelling method that has been employed in numerous software packages. This procedure helps nonexpert users to capture high-quality models rapidly and conveniently from uncalibrated images taken with an ordinary camera, without any advanced equipment or specially designed lighting conditions.

A phone camera and a personal computer with a moderate graphics card are good enough to process $3 \mathrm{D}$ models of small objects and artefacts from 
photographs. Nevertheless, digitising large-scale buildings or landscapes with thousands of photos may require significant hardware support and processing time. If the user has limited hardware, has a weaker graphics card (CUDA capable NVIDIA video card), or is working with large data sets, he/she might need to seek the help of cloud computing. Examples of commercial services supporting large data processing (including photo captured from drones/UAVs suitable for architecture, engineering, and construction [AEC] and survey industries) include:

- Autodesk ReCap Pro (https://www.autodesk.com/products/recap/)

- Get3d (www.get3d.cn)

- Pix4Dcloud (https://www.pix4d.com/product/pix4dcloud)

There is also non-commercial free cloud processing support for remote $3 \mathrm{D}$ reconstruction, such as ARC 3D Webservice (https://www.arc3d.be/). For cloud-based processing, users can upload photos/data set through a client tool and get informed by an email sent automatically from the server when the process is done, and the model is ready to download. An experienced user with some technical expertise can also 'rent' virtual machines (called cloud computing instances or instances) from commercial services like Amazon Web Service (AWS), Microsoft Azure, and Google Cloud for having more customised and robust performance. These virtual machines have the necessary power to process large photogrammetry projects. However, these commercial services come with various subscription model tiers.

Smartphones can be used for scanning and extrapolating a 3D model from an existing object with the aid of applications. These apps use a phone camera to scan an object and can process the data either within the phone or can send the data to an online server for remote processing. Examples of such applications include Qlone, Scandy Pro, Scann3D, Capture:3D Scan Anything, Trino, and Sony 3D Creator. Models generated from such an app may be less accurate than models derived from 3D scanners. Still, it is a great and affordable way to learn about 3D scanning without investing in high-end hardware and software.

There are some other hardware-based solutions for 3D scanning and modelling, such as Occipital Structure Sensor (https://structure.io/) connected via an iPad. A specific app allows 3D scanning, processing, and exporting (via email) the $3 \mathrm{D}$ model to specific formats and locations to support the device. The software is free to use.

We can also see some other emerging technologies and recent hardware development, assisting 3D scanning tasks. For example, the recent inclusion of a lidar scanner on the rear camera of the iPad Pro/iPhone 12 Pro looks promising and may help in mapping the environment and related 3D objects. Apple's lidar scanner is not yet at the level of professional lidar scanners used for outdoor surveying and scanning. However, it can still measure the distance to surrounding objects up to approximately five meters away, and it works both indoors and outdoors, at a photon level of nanoseconds. It can also track the location of people (spatial tracking) (Narain, 2020). A portable 3D laser scanner (such as 
FARO Freestyle 2) can also support fast and photorealistic 3D capture. The lightweight handheld device has a high degree of flexibility, which allows its operator to scan various places with challenging accessibility and conditions.

In general, the $3 \mathrm{D}$ reconstruction process comprises two parts: data acquisition and data processing. Data acquisition can be made through various hardware ranging from mobile phone cameras to satellite imaging. The data processing/3D reconstruction, on the other hand, can be undertaken with a mobile device, a local PC, or with the support of cloud-based computation. However, the selection of the hardware and software largely depends on the size and location of the object, allocated budget, technical expertise, and access to the service.

Image-based 3D modelling technique can be executed either in a local computer or in a remote computer/cloud server based on the adopted software/service. Software or services such as ARC3D and Autodesk Remake use the power of cloud computing to carry out the data processing in a remote server. By contrast, software including Regard3D, Aspect3D, Metashape, 3D SOM Pro, 3DF Zephyr, and so on process the data on a local (client) machine. This chapter does not cover the cloud processing method; instead, it presents a workflow solely featuring software/applications that run on a local computer or workstation.

\section{Photogrammetry}

Photogrammetry is all about 'measurement' of the subject of the image. A photographer needs to follow a specific rule-based procedure to collect the data set (photographs) to get a high-quality photogrammetric measurement. This standard photographing procedure, therefore, guides the photographer about configuring the camera, selection of certain objects, shooting techniques (overlapping), and positioning and orientation of the camera towards the subject in such a way that the processing software can minimise the uncertainty and produce the best result. A 3D model (or point clouds) can only be achieved when the relations between photographs are appropriately established. The success of such photogrammetry-based 3D modelling largely depends on the photographic data and the underlying algorithm of the processing and measuring software.

This 3D documentation is of prime importance in the fields of historic preservation, tourism, educational, and real state (Bertocci, Arrighetti \& Bigongiari 2019; Dhonjua et al. 2017). Image-based reconstruction or photogrammetry process claims to be cost-effective as compared to traditional laser scanning methods and can provide an automated system with considerable accuracy in the 3D model generation (Scianna \& La Guardia 2019; Skarlatos \& Kiparissi 2012). However, there are expenses to be incurred in acquiring commercial software licenses, and a certain level of technical skill and knowledge is essential.

Several articles have described the overall production of $3 \mathrm{D}$ models as a sequence of calibrated or uncalibrated photographs (Fuhrmann, Langguth 
\& Goesele 2014; Schöning \& Heidemann 2015; Skarlatos \& Kiparissi 2012), including details of different techniques for achieving a high degree of accuracy (Bolognesi et al. 2014; Nguyen \& Dang 2017).

One can also find comprehensive studies on image-based modelling software that analyses their performance (Durand, Engberg, \& Pope 2011; Grussenmeyer \& Al Khalil 2008; Scianna \& La Guardia 2019; Wang 2011), accuracy in 3D production (Bolognesi et al. 2014; Deseilligny, Luca \& Remondino 2011; Oniga, Chirilă \& Stătescu 2017), algorithms used (Knapitsch et al. 2017), and scalability (Knapitsch et al. 2017; Santagati, Inzerillo \& Di Paola 2013). A few studies also address workflows as well (Hafeez et al. 2016; Koutsoudis et al. 2008). However, research on either best practice or automated workflows that can be adopted by the general public to create free and easy $3 \mathrm{D}$ reconstruction models are hard to find, and low-cost workflows are scarce.

\section{Photogrammetry-Based 3D Modelling Workflow}

\section{Photography/Data Collection}

Photographs can be acquired from several cameras or a single camera. Uncalibrated photographs taken from general cameras are usually used to create a $3 D$ point cloud by image-based 3D reconstruction software. The software determines the geometric properties of the objects based on the provided photographs and some specific properties of the camera, such as the focal length and sensor size. This process requires comparing and referencing points or matching pixels across a series of photographs. Indeed, the quality and number of photographs play a vital role in allowing the algorithm to process the surface, match points, and triangulate the visual features. A successful compilation of the previous steps can then generate the $3 \mathrm{D}$ point cloud.

The camera can range from a mobile phone camera to a professional DSLR camera for photo shooting. Photos must be taken with the right amount of overlap while repositioning the camera for every shot. Depth of field is recommended as deep as possible (F.8 or higher) so more objects are in focus. The photos should not be over- or underexposed, as dark shadows and washed-out lighting can mask important details when processed. Shadows on an object may be mistaken by the photogrammetry software and can produce holes in the object, which will eventually be reflected in the resulting reconstruction. For best results, diffused light is preferred to avoid overexposure.

Background also plays a vital role in the output quality of the $3 \mathrm{D}$ model. The reconstruction may appear wrapped if the background prevents the object from being identified or distinguished from the surfaces or objects behind the subject. Objects with a glossy surface, uniform colour or texture, and transparent or translucent material will not yield a quality reconstruction. These objects generally produce holes in the resulting meshes or extensive warping. A 
mesh is a discrete representation of a geometric model in terms of its geometry, typology, and associated attributes (Comes, Buna \& Badiu 2014). Therefore, it is often recommended to avoid dark surfaces, reflective surfaces, transparent surfaces (including water), uniform textures and solid colour surfaces, moving light sources and shadows, and capturing the photographer's own shadow.

Generally, the object of interest should take up 70\% of the frame or more. Photographs should overlap every 5-10 degrees while capturing an object. The rule of thumb is, 'The more your photos overlap, the better your reconstruction'. Therefore, at least $50 \%$ of a photo needs to overlap with another. The more photos are captured, the greater chance of having an accurate model. For more information about image acquisition, please see Lab (2018).

\section{Data Processing/3D Model Development}

Structure from motion (SfM) is one of the most popular methods and often been recommended by many authors for 3D reconstruction (Nikolov \& Madsen 2016). Ranging from simple home-brew systems to high-end professional packages, there are a wide variety of $3 \mathrm{D}$ modelling programs available based on SfM in the market. Rahman and Champion (2019) present a comparison study of four popular free and open-source software (FOSS) packages that use the SfM techniques. This study, however, generalises the photogrammetric workflow as a six-step process to produce $3 \mathrm{D}$ reconstructions and $3 \mathrm{D}$ models, which includes the following:

(1) Add photos (or image acquisition),

(2) Align photos (or feature detection, matching, triangulation),

(3) Point cloud generation (or sparse reconstruction, bundle adjustment),

(4) Dense cloud generation (or dense correspondence matching),

(5) Mesh/surface generation, and

(6) Texture generation (a few software packages also offer cloud/mesh editing, such as Metashape).

Data/photo sets must be imported to the software first. Usually, users are given options to add/import the photos all at once or as a group (or chunk). The software analyses each photo through an algorithm (such as A-KAZE) and detects features (sometimes also called key points). 'Features' are points in an object that have a high chance of being found in different images of the same object, for example, edges, corners, marks, and the like.

For each feature, a mathematical descriptor is calculated. This descriptor has the characteristic that can identify the same point in an object in different images (seen from different viewpoints). Techniques such as LIOP (local intensity order pattern) can be used for this purpose. The descriptors from different images are then matched and geometrically filtered. This process results in a collection of matches between each image pair. 

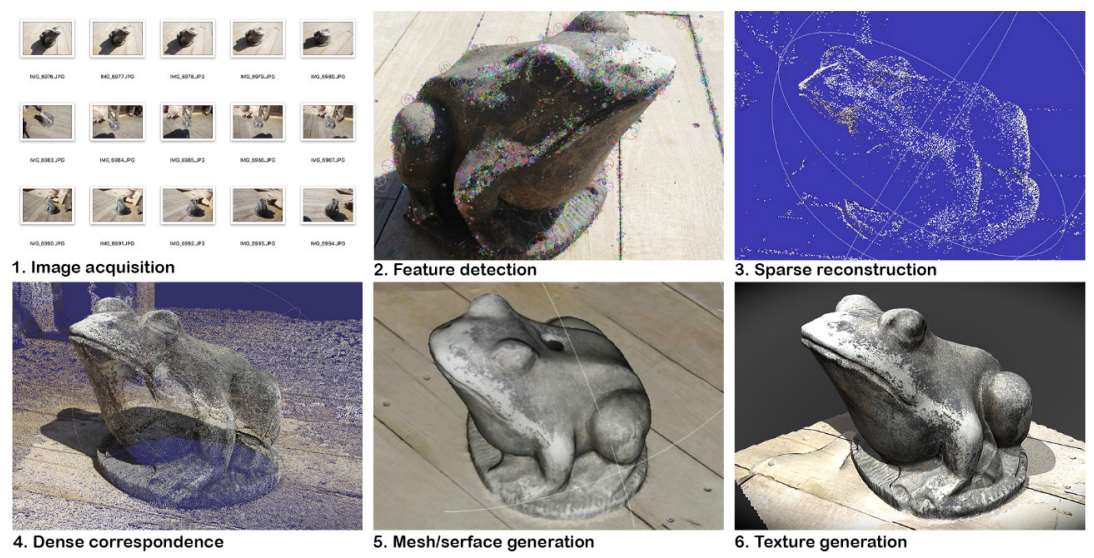

Figure 3: A typical workflow of photogrammetry data processing

(3D reconstruction).

The next is the calculation of the 'tracks.' Each feature that is part of a match in an image pair is also searched for in other images. A track is then generated from features if these features satisfy certain conditions, for example, if a track is seen in at least three images. Then it goes to the 'triangulation' phase. Matches from all the image pairs are then used to calculate the $3 \mathrm{D}$ position and characteristic of the 'camera'. In this enormous process, the algorithm finds the location where each image was shot, including the visual characteristics of the camera, followed by calculating the $3 \mathrm{D}$ position of each track.

The triangulation phase produces a sparse point cloud. To obtain a more dense point cloud or 'densification', several other algorithms are used. Finally, it comes to surface generation. The point clouds are used to generate a surface, either with coloured vertices or with texture. However, not all photogrammetry software offers mesh editing, mesh optimising, cap holes, and texture generation. For a better result, additional software, with some additional steps, may need to be used.

\section{Further Improvement/Mesh Generation and Editing}

Photogrammetry software usually generates noise, outliers, and irrelevant points while developing the point clouds, and the result requires cleaning. Not all packages support editing and cleaning. The creation of a mesh from uncleaned point clouds is not recommended, as it will require more time for computation and eventually will generate unwanted surfaces. Once the mesh is developed from the point clouds, software packages such as 3D Studio Max, Maya, ZBrush, Blender, and MeshLab can be used for further processing. 

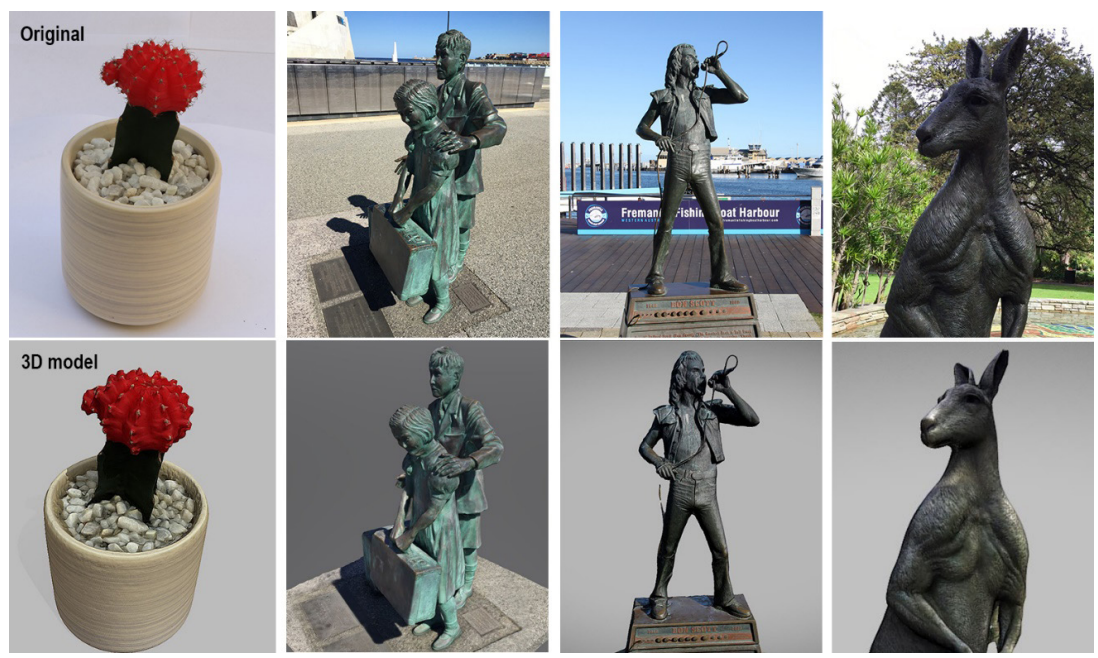

Figure 4: Original versus 3D reconstruction of objects.

The generated point clouds can be imported directly to MeshLab (http: //meshlab.net), which is free and open-source software (FOSS). MeshLab provides various tools for selection and removal of points/vertexes (noise, cleaning outliers), surface reconstruction (or mesh generation), simplification of mesh, and 'cap hole' (or close holes tools closes void areas on the model substituting photogrammetric reconstruction with extrapolation data) steps that enhance the $3 \mathrm{D}$ model.

Mesh simplification is self-explanatory. It decreases the number of polygons while keeping the shape as close as possible to the original. Because of the reduction of the poly count, processing is faster in $3 \mathrm{D}$ visualisation or in a game engine. Cap holes close or cover any gap or hole where the previous mesh generation failed to generate a polygon.

'Texturing' provides visual skin/membrane coverage for the $3 \mathrm{D}$ models, so that the virtual objects resemble the original and look realistic. MeshLab can export a wide range of $3 \mathrm{D}$ file formats with supporting textures (e.g., ${ }^{\star} . x 3 \mathrm{~d}$, ${ }^{\star}$.obj), including 3D point cloud with vertex or points colour $\left({ }^{*}\right.$.ply).

\section{Applications and Possibilities}

The application of photogrammetry covers the broad areas of topographic mapping, architecture, engineering, manufacturing, quality control, police investigation, cultural heritage, geology, and the film and game industries.

Virtual heritage, digital preservation, and archiving: Archaeology and cultural heritage both use photogrammetry for digital preservation of heritage assets 


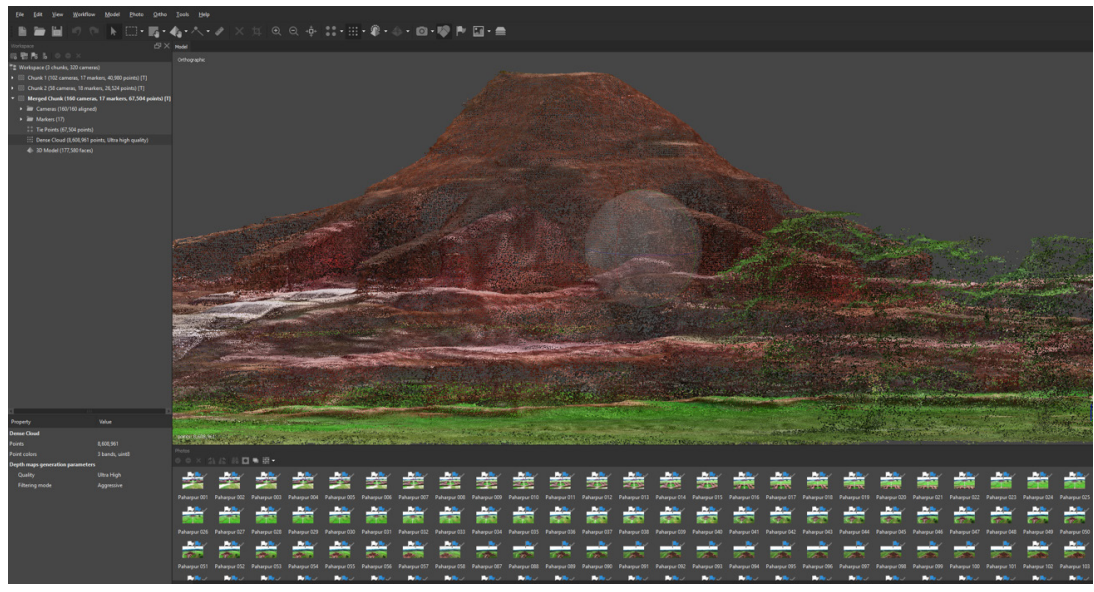

Figure 5: Dense point cloud generated from photographs (Sompur Mahavihara, Bangladesh, a world heritage site) using photogrammetry.

and sites. There is a remarkable number of public, commercial, and hobbyist 3D repositories for hosting and archiving 3D heritage models. CARARE, Europeana, Smithsonian, TurboSquid, Sketchfab, and the like support hosting and archiving 3D heritage assets. There are more than fifty commercial repositories, such as ShareCG, Turbo Squid, Blendswap, and MyMiniFactory. However, they are mostly commercial marketplaces and do not necessarily support 3D archiving.

Land surveying: Photogrammetry and land surveying have many similarities, and that is why this technique has become popular in this field. Besides satellite imagery, drones are getting popular as a cheaper alternative for obtaining images of contours and land masses. Land surveyors and building professionals can utilise photogrammetry for automated generation of $3 \mathrm{D}$ surface models, developing rectified and orthorectified imagery and localised mapping from photography gathered by a UAV (drone).

Forensic and accident reconstruction: Photogrammetry has been used successfully for crime scene investigation, forensics, and traffic accident reconstruction and measurement. The technology is equally applicable for both indoors and outdoors. Recording tiny details are essential in these cases, and photogrammetry can help in a court of law by documenting the precise measurements of the crime scene.

Filmmaking: Filmmakers have relied on 3D modelling and 3D animations for decades. Photogrammetry is now supporting this demand by providing a more realistic and accurate $3 \mathrm{D}$ environment. Close-range digital photogrammetry can be used in developing accurate 3D movie sets, while the location scouting can be done virtually using aerial photogrammetry. Photogrammetry can also be used in filmmaking for creating digital stunt doubles, texture mapping, crowd replication, and more. 
$3 D$ assets and environments for games: Photogrammetry is being used in video games to create high-quality $3 \mathrm{D}$ assets and $3 \mathrm{D}$ realistic environments. The developers of "The Vanishing of Ethan Carter" first applied this technology in 2014 for creating stunning settings for the game. Since then, photogrammetry has been extensively used for 3D.

Building design and renovation: Building renovation requires drawings as a reference point for decision making for architects and associated professionals. However, construction drawings are often rarely found for historic buildings. In these cases, photogrammetry software can be used to create point cloud and 3D models, which can later be converted to CAD drawings and BIM (Building Information Modelling). Photogrammetry software is equally useful in surveying the surrounding area for further use in landscape design.

Geology and mining: Disciplines such as geology and mining use photogrammetry for various applications. Photogrammetry provides affordable solutions in generating point clouds and elevation maps, which can be used as comprehensive analytical tools to measure stockpiles and pits instantly.

$3 D$ printing and prototyping: Industries including the automobile, jewellery, and medical training industries use photogrammetry and 3D printing. Instead of creating an object manually, photogrammetry can transform images into 3D models and can cut down the required time in model production. Once the model is captured from the real-world object, it can be reproduced at any scale (Lima et al. 2019).

Photogrammetry in sports: Close-range photogrammetry is used for evaluation of players' performance and in developing virtual training systems. A photogrammetric system can track and measure the most accurate body movement of an expert player such as a javelin thrower's shooting motion. These parameters then can be used to train new players. Analysing payers' movement (biomechanical differences) also allows the scientists and athletes to minimise adverse body effects, such as an improper movement of the elbow joint during the slap-shot of ice hockey athletes (Caniberk, Sesli \& Çetin 2017).

\section{Conclusion}

Due to the recent concerns regarding the destruction and damage inflicted on internationally recognised heritage sites in Afghanistan, Syria, Iraq, and most recently in Brazil and France, digital documentation and 3D preservation of historical and monuments have progressively entered the arena of international importance. Thanks to emerging computer technologies and the advancements in photogrammetry software, which is helping us to streamline the process of $3 \mathrm{D}$ documentation and digital conservation. In particular, the development of affordable techniques such as image-based photo modelling and free and open-source software (FOSS) is undeniable. The rapid adoption 
and application of photogrammetry in domains other than architecture and archaeology is also remarkable.

The process of image-based modelling is easy; however, photo shooting for this purpose requires specific skills and following certain rules. The software can only produce the 3D model (or part of it) if it is visible to the camera. This means any unseen section in the photograph will produce holes in the model. Not all objects are suitable for image-based photo modelling. More photos can produce better 3D models, but at the same time, more data sets require more powerful hardware and graphic card support. Free and open-source software is not always bug-free, and the developer rarely provides technical support. Regardless of these limitations, photogrammetry or image-based 3D modelling is a preferable solution for digital documentation and preservation of heritage and cultural artefacts, buildings, and sites.

\section{References}

Bertocci, S, Arrighetti, A, and Bigongiari, M 2019 Digital survey for the archaeological analysis and the enhancement of Gropina Archaeological Site. Heritage, 2(1): 848-857. DOI: https://doi.org/10.3390/heritage2010056

Bolognesi, M., Furini, A., Russo, V., Pellegrinelli, A., and Russo, P 2014 Accuracy of cultural heritage 3D models by RPAS and terrestrial photogrammetry. In Proceedings of The International Archives of the Photogrammetry, Remote Sensing and Spatial Information Sciences, Riva del Garda, Italy, (pp. 113-119). DOI: https://doi.org/10.5194/isprsarchives-XL-5-113-2014

Comes, R, Buna, Z, and Badiu, I (2014). Creation and preservation of digital cultural heritage. Journal of Ancient History and Archaeology, 1(2): 50-56. DOI: https://doi.org/10.14795/j.v1i2.55

Deseilligny, M P, Luca, L D, and Remondino, F 2011 Automated image-based procedures for accurate artifacts 3D modelling and orthoimage generation. Geoinformatics FCE CTU, 6: 291-299. DOI: https://doi.org/10.14311 /gi.6.36

Dhonjua, H, Xiaob, W, Sarhosis, V, Mills, J, Wilkinson, S, Wang, Z, Thapa, L, and Panday, U 2017 Feasibility study of low-cost image-based heritage documentation in Nepal. International Archives of the Photogrammetry; Remote Sensing and Spatial Information Sciences: Göttingen, Germany, 237-242.

Durand, H, Engberg, A, and Pope, S T 2011 A comparison of $3 d$ modeling programs. Santa Barbara, USA.

Fuhrmann, S, Langguth, F, and Goesele, M 2014, 6-8 Oct MVE-A multi-view reconstruction environment. In EUROGRAPHICS Workshops on Graphics and Cultural Heritage, (pp. 11-18) Darmstadt, Germany. DOI: https://doi .org/10.2312/gch.20141299

Grussenmeyer, P, and Al Khalil, O 2008 A comparison of photogrammetry software packages for the documentation of buildings. Paper presented at The Mediterranean Surveyor in the New Millennium. Malta. 
Hafeez, J, Hamacher, A, Son, H-J, Pardeshi, S, and Lee, S-H 2016 Workflow evaluation for optimized image-based $3 \mathrm{D}$ model reconstruction. In the Proc. of International Conference on Electronics, Electrical Engineering, Computer Science (EEECS): Innovation and Convergence, (pp. 62-65).

Knapitsch, A, Park, J, Zhou, Q-Y, and Koltun, V 2017 Tanks and temples: Benchmarking large-scale scene reconstruction. ACM Transactions on Graphics (TOG), 36(4), 78. DOI: https://doi.org/10.1145/3072959.3073599

Koutsoudis, A, Arnaoutoglou, F, Pavlidis, G, Tsiafakis, D, and Chamzas, C 2008 A versatile workflow for 3D reconstructions and modelling of cultural heritage sites based on open source software. In Proceedings of Virtual Systems and Multimedia Dedicated to Digital Heritage Conference, Limassol, Cyprus, (pp. 238-244).

Lab, D 2018. Getting started: A guide to photogrammetry. 3D Capturing Technology, 16. Retrieved from University of Michigan 3D Lab website: http: //um3d.dc.umich.edu/wp-content/uploads/2017/01/Photo_Capturing_for _Photogrammetry-1.pdf.

Lima, L F S d, Barros, A J B P d, Martini, A d C, Stocco, M B, Kuczmarski, A H, and Souza, R L d 2019 Photogrammetry and 3D prototyping: A lowcost resource for training in veterinary orthopedics. Ciência Rural, 49(12). DOI: https://doi.org/10.1590/0103-8478cr20180929

Narain, A. 2020. Apple's LiDAR Scanner a game-changer in scanning technology? Retrieved from https://www.geospatialworld.net/blogs/apples-lidar -scanner/.

Nguyen, M H, Wünsche, B, Delmas, P, and Lutteroth, C 2012, June 25-28 3D models from the black box: Investigating the current state of image-based modeling. In 20th International Conference on Computer Graphics, Visualisation and Computer Vision (WSCG 2012)) Pilsen, Czech Republic.

Nguyen, V T, and Dang, T 2017 Setting up virtual reality and augmented reality learning environment in Unity. In 2017 IEEE International Symposium on Mixed and Augmented Reality (ISMAR-Adjunct), (pp. 315-320).

Nikolov, I A, and Madsen, C B 2016, 31 Oct-5 Nov Benchmarking close-range structure from motion 3D reconstruction software under varying capturing conditions. In 6th International Conference, EuroMed 2016, (pp. 15-26) Nicosia, Cyprus.

Oniga, E, Chirilă, C, and Stătescu, F 2017, 1-3 March Accuracy assessment of a complex building 3D model reconstructed from images acquired with a lowcost UAS. In ISPRS-International Archives of the Photogrammetry, Remote Sensing and Spatial Information Sciences, (pp. 551-558) Nafplio, Greece.

Rahaman, H, and Champion, E 2019 To 3D or not 3D: Choosing a photogrammetry workflow for cultural heritage groups. Heritage, 2(3): 1835-1851. DOI: https://doi.org/10.3390/heritage2030112

Santagati, C, Inzerillo, L, and Di Paola, F 2013, 2-6 Sept Image-based modeling techniques for architectural heritage 3D digitalization: Limits and 
potentialities. In International Archives of the Photogrammetry, Remote Sensing and Spatial Information Sciences, XXIV International CIPA Symposium, Strasbourg, France.

Schöning, J, and Heidemann, G 2015, 22-24 Aug Evaluation of multi-view 3D reconstruction software. In International Conference on Computer Analysis of Images and Patterns, (pp. 450-461) Valletta, Malta.

Scianna, A, and La Guardia, M 2019 Survey and photogrammetric restitution of monumental complexes: Issues and solutions-The case of the Manfredonic Castle of Mussomeli. Heritage, 2(1): 774-786. DOI: https://doi .org/10.3390/heritage 2010050

Skarlatos, D, and Kiparissi, S 2012 Comparison of laser scanning, photogrammetry and SFM-MVS pipeline applied in structures and artificial surfaces. ISPRS Annals of the Photogrammetry, Remote Sensing and Spatial Information Sciences, 3: 299-304.

Wang, Y-F 2011 A Comparison Study of Five 3D Modeling Systems Based on the SfM Principles. Retrieved from http://www.visualsize.com/. 